
Military Technical College Kobry El-Kobbah Cairo, Egypt

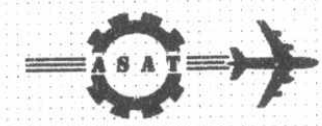

$10^{\text {th }}$ International Conference On Aerospace Sciences\& Aviation Technology

\title{
MATRIX FORMULATION OF CHEBYSHEV SOLUTION TO SHELL PROBLEMS
}

\author{
A. OKASHA EL-NADY ${ }^{1}$ \\ HANI M. NEGM ${ }^{2}$
}

\begin{abstract}
Any continuous function $f(\xi)$ can be expanded in a Chebyshev series. The $n^{\text {th }}$ derivative of the function $f(\xi)$ can be written in matrix form in terms of the expansion coefficients of the function. Also, the product of two functions $f(\xi)$ and $g(\xi)$ can be written in matrix form in terms of the expansion coefficients of the two functions. Therefore, any system of differential equations with variable coefficients can be written as a system of algebraic equations in terms of Chebyshev coefficients of the functions, which can be easily solved. The method is used to solve the problem of isotropic conical shell with different loads and boundary conditions. Results are computed and compared with the exact ones. Comparison proves convergence, accuracy and reliability of the proposed method.
\end{abstract}

\section{KEYWORDS}

Boundary-value problems. Differential equations. Chebyshev series. Shells. Conical shells.

\section{NOMENCLATURE}

$$
\begin{aligned}
& \mathrm{C}=\frac{E h}{1-v^{2}} \\
& \mathrm{D}=\frac{E h^{3}}{12\left(1-v^{2}\right)}
\end{aligned}
$$

\footnotetext{
${ }^{1}$ Aerospace Research Center, AOI

${ }^{2}$ Aerospace Eng. Dept., Cairo University.
} 


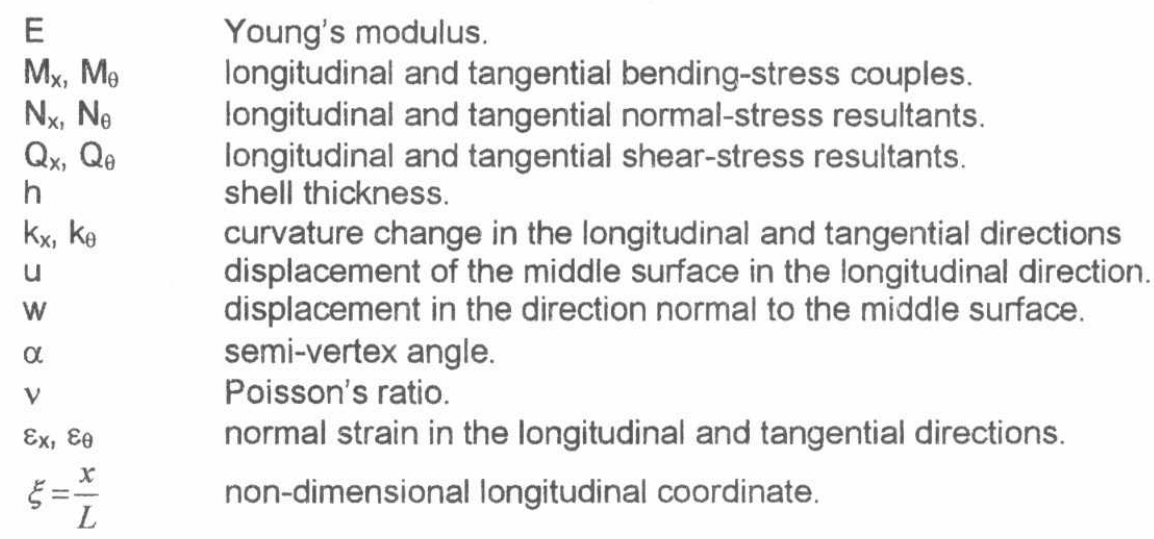

\section{INTRODUCTION}

Applications of shells or shell-like structures as load-bearing members are common in aerospace, automobile and other industries due to their efficiency as structural components [1]. A considerable amount of research has been done on the development of new shell theories as well as the solution of their equilibrium differential equations. The solution of differential equations has two different approaches: numerical and analytical. Analytical solutions are either exact or approximate.

Exact solutions may be easily obtainable in case of shells with simple geometry and boundary conditions, and uniform thickness and elastic properties. In many circumstances, however, it is not possible to find suitable functions which satisfy both the shell governing differential equations and the geometric and natural boundary conditions [2].

Geckeler [3] suggested an approximate analytical method for the solution of boundary-value problems of thin bending-resistant shells. The Asymptotic Integration method [4] is another approximate analytical method for the solution of shell problems.

Many approximate numerical techniques for the solution of boundary-value problems are available. The finite-difference method [5-7], the finite-element method [8,9] and numerical-integration methods $[10,11]$ are examples of these numerical techniques.

A suitable solution function that is able to satisfy many differential equations and boundary conditions is the Chebyshev polynomial. Alwar and Narasimhan [12, 13] used Chebyshev polynomials to solve the problem of spherical shell under axisymmetric and general loads.

The objective of this paper is to reformulate the Chebyshev series technique in matrix form to make it easier, more reliable and less time consuming. Using matrix notation, the function derivatives and function products can be represented in Chebyshev 
series in a straightforward and simple manner. This arrangement converts ordinary differential equations with variable coefficients into a simple system of algebraic equations.

Two examples of conical shells with different boundary conditions are worked out in this paper to illustrate the application of the suggested matrix formulation of the Chebeyshev technique to shell problems. The first example deals with an isotropic complete cone fixed at its base under uniform internal pressure. The second example deals with an isotropic frusrtum hinged at its base under concentrated lateral and edge line loads. Results are compared to those obtained by other techniques to demonstrate the accuracy and reliability of the suggested technique.

\section{CHEBYSHEV SERIES REPRESENTATION}

Any continuous function $f(\xi)$ in the interval $0 \leq \xi \leq 1$ can be written in Chebyshev series as follows [12, 13]:

$\mathrm{f}(\xi)=\sum_{r=0}^{\infty}{ }^{+} a_{r} T_{r}(\xi)$

where:

+ sign means that the $1^{\text {st }}$ term must be halved,

$a_{r} \ldots$ are constants to be determined so as to obtain the best possible fit.

$$
T_{r}(\xi)=\operatorname{Cos}(r t), \quad \operatorname{Cos}(t)=2 \xi-1, \quad 0 \leq \xi \leq 1
$$

The shifted Chebyshev polynomials satisfy the recurrence relations:

$T_{r+1}(\xi)=2(2 \xi-1) T_{r}(\xi)-T_{r-1}(\xi) \quad, 2 \leq r \leq \infty$

$T_{0}=1, \quad T_{1}=2 \xi-1$

And the orthogonality conditions:

$\int_{0}^{1} \frac{T_{m}(\xi) T_{n}(\xi)}{\sqrt{\xi} \sqrt{1-\xi}} d \xi=\left\{\begin{array}{llc}0 & \text { for } & m \neq n \\ \frac{\pi}{2} & \text { for } & m=n \neq 0 \\ \pi & \text { for } & m=n=0\end{array}\right.$

For any continuous function $f(\xi)$ the series expansion (1) is fast converging, and a good approximation is obtained by taking a finite number of terms. Therefore, equation (1) is approximated by:

$\mathrm{f}(\xi)=\sum_{r=0}^{N}{ }^{+} a_{r} T_{r}(\xi)$ 
where, for a known function $f(\xi)$, the coefficients ar are given by:-

$a_{r}=\frac{2}{\pi} \int_{0}^{1} \frac{f(\xi) T_{r}(\xi)}{\sqrt{\xi} \sqrt{1-\xi}} d \xi \quad 0 \leq \mathrm{r} \leq \mathrm{N}$

The first derivative $f^{\prime}(\xi)$ is expressed in Chebyshev series as $[12,13]$ :

$f^{\prime}(\xi)=\sum_{r=0}^{N-1}{ }^{+} a_{r}^{(1)} T_{r}(\xi)$

The coefficients $a_{r}^{(1)}$ satisfy the recursive relation:

$a_{r-1}^{(1)}-a_{r+1}^{(1)}=4 r a_{r} \quad, \quad 1 \leq \mathrm{r} \leq \mathrm{N}$

Similarly the higher derivatives can be written as:

$f^{\prime \prime}(\xi)=\sum_{r=0}^{N-2}+a_{r}^{(2)} T_{r}(\xi)$

$\mathrm{f}^{\mathrm{m}}(\xi)=\sum_{r=0}^{N-m}{ }^{+} a_{r}^{(m)} T_{r}(\xi)$

where;

$a_{r-1}^{(2)}-a_{r+1}^{(2)}=4 r a_{r}^{(1)}, \quad 1 \leq \mathrm{r} \leq \mathrm{N}-1$

$a_{r-1}^{(m)}-a_{r+1}^{(m)}=4 r a_{r}^{(m-1)}, \quad 1 \leq \mathrm{r} \leq \mathrm{N}-(\mathrm{m}-1)$

\section{MATRIX REPRESENTATION OF FUNCTION DERIVATIVES:}

The first-order-derivative coefficients $\left\{a_{r}^{(1)}\right\}$ in equation (6) can be written in terms of the original function coefficients $\left\{a_{i}\right\}$ using matrix notation as follows:

$\left\{a_{r}^{(1)}\right\}=4[\mathrm{~A}]\left\{\mathrm{a}_{i}\right\} \quad ; \quad \begin{aligned} & \mathrm{r}=0,1,2, \ldots \ldots \ldots \ldots, \mathrm{N}-1 \\ & \mathrm{i}=1,2,3 \ldots \ldots \ldots \ldots \ldots, \mathrm{N}\end{aligned}$

where $[A]$ is an upper triangular matrix of order $\mathrm{N} \times \mathrm{N}$.

The elements of the matrix $a_{i j}$ are defined as:

$$
\mathrm{a}_{\mathrm{ij}}=\left\{\begin{array}{lllll}
0 & i>j & \text { Or } & i+j & \text { odd } \\
j & i \leq j & \text { and } & i+j & \text { even }
\end{array}\right.
$$


The form of $[A]$ for $N=5$ for example is: $\quad A=\left[\begin{array}{lllll}1 & 0 & 3 & 0 & 5 \\ 0 & 2 & 0 & 4 & 0 \\ 0 & 0 & 3 & 0 & 5 \\ 0 & 0 & 0 & 4 & 0 \\ 0 & 0 & 0 & 0 & 5\end{array}\right]$

From equation (8), it is noted that the first-order-derivative coefficients are written in terms of the $\mathrm{N}$ coefficients $\left\{a_{i}\right\}(i=1,2, \ldots, N)$ of the function $f(\xi)$. To represent $\left\{a_{r}^{(1)}\right\}$ in terms of all function coefficients $\left\{a_{i}\right\}, i=0,1,2, \ldots \ldots, N$ we add a new left column with zero entries in the matrix [A], and the new matrix is termed [A01]. Thus:
$\left\{a_{r}^{(1)}\right\}=4[\mathrm{A01}]\left\{\mathrm{a}_{1}\right\}$
$r=0,1,2$,
$\mathrm{i}=0,1,2,3$
$\mathrm{N}-1$
$\mathrm{N}$

where [A01] is of order $\mathrm{N} \times \mathrm{N}+1$.

For $N=5$ : [A01] take the form: [A01] $=\left[\begin{array}{llllll}0 & 1 & 0 & 3 & 0 & 5 \\ 0 & 0 & 2 & 0 & 4 & 0 \\ 0 & 0 & 0 & 3 & 0 & 5 \\ 0 & 0 & 0 & 0 & 4 & 0 \\ 0 & 0 & 0 & 0 & 0 & 5\end{array}\right]$

The second-order-derivative coefficients $\left\{a_{r}^{(2)}\right\}$ in equation (7) can be written in terms of the function coefficients $\left\{a_{i}\right\}$ using matrix notation as follows:

$\left\{a_{r}^{(2)}\right\}=16[\mathrm{~A}]_{-1,-1}^{-1}[\mathrm{A01}]\left\{\mathrm{a}_{\mathrm{i}}\right\}$

$\left\{a_{r}^{(2)}\right\}=16[\mathrm{AO} 2]\left\{\mathrm{a}_{\mathrm{i}}\right\}$

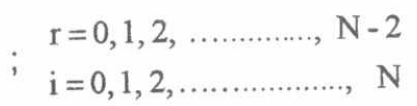

The third-order-derivative coefficients $\left\{a_{r}^{(3)}\right\}$ can be written in terms of the function coefficients $\left\{a_{1}\right\}$ using matrix notation as follows:

$$
\begin{aligned}
& \left\{a_{r}^{(3)}\right\}=64[\mathrm{~A}]_{-2,-2}^{-1}[\mathrm{AO2}]\left\{\mathrm{a}_{i}\right\} \\
& \left\{a_{r}^{(3)}\right\}=64[\mathrm{AOO}]\left\{\mathrm{a}_{\mathrm{i}}\right\} \\
& ; \begin{array}{l}
r=0,1,2, \ldots \ldots \ldots \ldots . ., \mathrm{N}-3 \\
\mathrm{i}=0,1,2, \ldots \ldots \ldots \ldots \ldots ., \mathrm{N}
\end{array}
\end{aligned}
$$

The general form of the $\mathrm{n}^{\text {th }}$-derivative coefficients $\left\{a_{r}^{(n)}\right\}$ can be written in terms of the function coefficients $\left\{a_{1}\right\}$ using matrix notation as follows:

$$
\begin{array}{ll}
\left\{a_{r}^{(n)}\right\}=(4)^{n}[A]_{1-n, 1-n}{ }^{-1}[A O(n-1)]\left\{a_{i}\right\} & \\
\left\{a_{r}^{(n)}\right\}=(4)^{n}[A O n]\left\{a_{i}\right\} & ; \quad \begin{array}{l}
r=0,1,2, \ldots \ldots \ldots \ldots, N-n \\
i=0,1,2, \ldots \ldots \ldots \ldots \ldots . . ., N
\end{array}
\end{array}
$$


where,

\section{New Form of Chebyshev Series}

Any continuous function $f(\xi)$ in the interval $0 \leq \xi \leq 1$ and its derivatives can be written in the new matrix form of Chebyshev series as follows:
$f(\xi)=\left[T_{r}\right][1]\left\{a_{i}\right\}$
$r=0,1,2, \ldots \ldots \ldots \ldots \ldots ., N$
$i=0,1,2, \ldots \ldots \ldots \ldots \ldots ., N$
$f^{n}(\xi)=(4)^{n}\left[T_{r}\right][A O n]\left\{a_{i}\right\}$

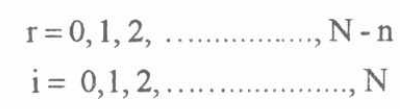

where; $\left[T_{r}\right]$ is a row matrix whose elements are $T_{r}(\xi)$. Note that in the new formulation the first term of $\left[T_{r}\right]$ must be halved.

Now consider the general nonhomogenous differential equation of $n^{\text {th }}$ order:

$f^{n}+f^{n-1}+f^{n-2}+$ $f^{\prime}+f=p$

After expanding each term in Chebyshev series the above differential equation can be written as:

$\sum_{r=0}^{N-n}{ }^{+} a_{r}^{(n)} T_{r}(\xi)+\sum_{r=0}^{N-(n-1)}+a_{r}^{(n-1)} T_{r}(\xi)+\ldots+\sum_{r=0}^{N-1}+a_{r}^{(1)} T_{r}(\xi)+\sum_{r=0}^{N}+a_{r} T_{r}(\xi)=\sum_{r=0}^{N}+p_{r} T_{r}(\xi)(12)$

The forcing-function coefficients $\mathrm{p}_{\mathrm{r}}$ can be evaluated using equation (4). Equating the coefficients of like Chebyshev polynomial terms on either side, the resulting $N+1-n$ algebraic equations can be written in matrix form using equations (10) and (11) as:

$$
\begin{aligned}
{\left[4^{(n)}[A O n]+4^{(n-1)}[A O(n-1)]+\ldots+4\right.} & {[A 01]+[1]]\left\{a_{i}\right\}=\left\{p_{r}\right\} } \\
& r=0,1,2, \ldots \ldots \ldots \ldots . ., N+1-n \\
i & =0,1,2, \ldots \ldots \ldots \ldots \ldots, N
\end{aligned}
$$

where all matrices in equation (13) are of the same order $(N+1-n \times N+1)$. For all derivatives lower than the highst derivative, the first $N+1-n$ rows are chosen so as to satisfy equation (13). In order to be able to solve equation (13), $n$ additional equations are needed. These additional equations are supplied by the problem boundary conditions. 


\section{MATRIX REPRESENTATION OF FUNCTION PRODUCTS}

If $f(\xi)$ and $g(\xi)$ are two continuous functions represented by truncated Chebysehev series as:

$f(\xi)=\sum_{r=0}^{N}+a_{r} T_{r}(\xi)$

$g(\xi)=\sum_{r=0}^{M}{ }^{+} b_{r} T_{r}(\xi)$

Then the product of these functions can be written in a Chebyshev series as:

$\mathrm{g}(\xi) \mathrm{f}(\xi)=\sum_{r=0}^{M+N}{ }^{+} c_{r} T_{r}(\xi)$

where

$$
\begin{aligned}
& c_{0}=\sum_{i=0}^{N+M}{ }^{+} a_{i} b_{i} \\
& \mathrm{c}_{\mathrm{r}}=\frac{1}{2} \sum_{i=0}^{N+M}{ }^{+} a_{i}\left(b_{i+r}+b_{|i-r|}\right) \quad ; \quad \begin{array}{c}
1 \leq r \leq M+N \\
i+r \leq M \\
|i-r| \leq M
\end{array} \quad \forall i
\end{aligned}
$$

The $\left\{c_{r}\right\}$ coefficients can be written in terms of the $\left\{a_{i}\right\}$ coefficients only using matrix notation as follows:

$$
\left\{c_{r}\right\}=[H]\left\{a_{i}\right\}
$$

where

$$
\begin{aligned}
& r=0,1,2, \ldots \ldots \ldots, N+M \\
& i=0,1,2, \ldots \ldots \ldots, N \\
& \left\{c_{r}\right\} \ldots \text { is a column matrix of order } N+M+1 \times 1 \\
& {[H] \ldots \text { is rectangular matrix of order } N+M+1 \times N+1} \\
& \left\{a_{i}\right\} \ldots \text { is a column matrix of order } N+1 \times 1
\end{aligned}
$$

The coefficients $\left\{b_{i}\right\}$ are obtained by forcing the function $g(\xi)$ to take on its true values at a number of selected points in the interval $0 \leq \xi \leq 1$. Hence $h_{i j}$ can be written as:

$$
\mathrm{h}_{\mathrm{ij}}=\left\{\begin{array}{lrl}
\frac{1}{4}\left(b_{|i-j|}+b_{i+j-2}\right) & \text { for } \begin{array}{l}
i=1,2, \ldots \ldots \ldots, N+M+1 \\
j=1
\end{array} & \begin{array}{l}
i-j \mid \leq M \\
i=1,2, \ldots \ldots \ldots, N+M+1
\end{array} \\
\frac{1}{2}\left(b_{|i-j|}+b_{i+j-2}\right) & \text { for } \begin{array}{l}
i+j-2 \leq M \\
j=2,3, \ldots \ldots \ldots, N+1
\end{array}
\end{array} \forall i, j\right.
$$


Therefore, equation (14) takes the form:

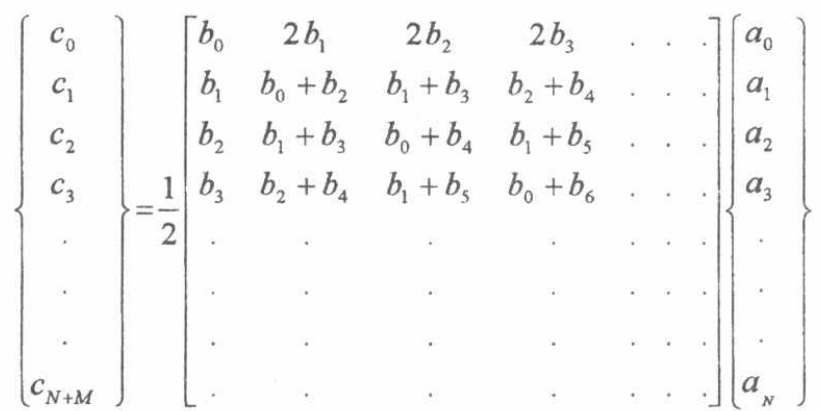

THE PROBLEM OF AXISYMMETRIC CONICAL SHELL WITH BENDING RESISTANCE:

\section{Equilibrium Equations}

The governing equations of a bending-resistant conical shell under normal pressure load $\mathrm{q}$ are given by [2]:

$\frac{d N_{x}}{d x}+\frac{1}{x}\left(N_{x}-N_{\theta}\right) \quad=0$

$\frac{d Q_{x}}{d x}+\frac{1}{x}\left(Q_{x}-N_{\theta} \cot \alpha\right)=q$

$\frac{d M_{x}}{d x}+\frac{1}{x}\left(M_{x}-M_{\theta}\right)-Q_{x}=0$

The shell geometry and stress resultants are shown in Figs. (1) and (2).

\section{Constitutive Relations}

The shell constitutive relations between stress resultants and stain and curvature components are given by [2]:

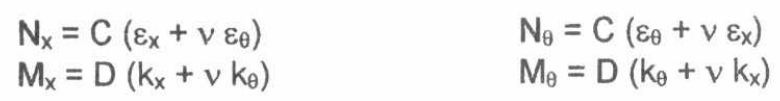

\section{Strain-Displacement Relations}

The shell strain-displacement relations for small displacements are given by [2]: 


$$
\begin{array}{ll}
\varepsilon_{\mathrm{x}}=\frac{d u}{d x} & \varepsilon_{\theta}=\frac{1}{x}(u+w \cot \alpha) \\
\mathrm{k}_{\mathrm{x}}=-\frac{d^{2} w}{d x^{2}} & \mathrm{k}_{\theta}=-\frac{1}{x} \frac{d w}{d x}
\end{array}
$$

Note that the stress resultants $N_{x \theta}, N_{\theta x}, M_{x \theta}, M_{\theta x}$, the stain components $\varepsilon_{x \theta}, k_{x \theta}$ and the displacement component $v$ in the tangential direction are all zero because of the axisymmetric nature of the problem.

Substituting the strain displacement-relations (17) into the stress resultant-strain relations (16), and eliminating $Q_{x}$ between the last two equations of (15) we end up with the following two equilibrium differential equations:

$$
\begin{aligned}
& \frac{C}{L^{2}}\left(\frac{d^{2} u}{d \xi^{2}}+\frac{1}{\xi} \frac{d u}{d \xi}-\frac{1}{\xi^{2}} u\right)+\frac{C \cot \alpha}{L^{2}}\left(\frac{v}{\xi} \frac{d w}{d \xi}-\frac{1}{\xi^{2}} w\right) \\
& \frac{C \cot \alpha}{L^{2}}\left(\frac{v}{\xi} \frac{d u}{d \xi}+\frac{1}{\xi^{2}} u\right)+\frac{D}{L^{4}}\left(\frac{d^{4} w}{d \xi^{4}}+\frac{2}{\xi} \frac{d^{3} w}{d \xi^{3}}-\frac{1}{\xi^{2}} \frac{d^{2} w}{d \xi^{2}}+\frac{1}{\xi^{3}} \frac{d w}{d \xi}+\frac{C \cot ^{2} \alpha}{D} \frac{1}{\xi^{2}} w\right)=p
\end{aligned}
$$

Expanding $u(\xi)$ and $w(\xi)$ in $(N+1)$-term Chebyshev series we have a total of $2 N+2$ unknown coefficients. The functions $\frac{1}{\xi}, \frac{1}{\xi^{2}}$ and $\frac{1}{\xi^{3}}$ appearing in the equilibrium equations are also expanded in Chebyshev series having $\mathrm{M}+1$ terms. The $\mathrm{M}+1$ expansion coefficients can be computed easily by forcing the functions $\frac{1}{\xi}, \frac{1}{\xi^{2}}$ and $\frac{1}{\xi^{3}}$ to take on their actual values at a number of chosen points in the interval $0 \leq \xi \leq 1$. Using matrix notation for the functions and function derivatives, and applying the rule of matrix multiplications, the equilibrium equations can be written as a system of algebraic equations in the following matrix form:

$$
\begin{array}{r}
\frac{C}{L^{2}}[16[A 02]+4[H 101]-[H 2]]\left\{u_{i}\right\}+\frac{C \cot \alpha}{L^{2}}[4 v[H 101]-[H 2]]\left\{w_{i}\right\} \quad=\{0\} \\
\frac{C \cot \alpha}{L^{2}}[4 V[H 101]+[H 2]]\left\{u_{i}\right\}+\frac{D}{L^{4}}[256[A 04]+128[H 103]-16[H 202]+4[H 301]+ \\
\left.+\frac{C L^{2} \cot ^{2} \alpha}{D}[H 2]\right]\left\{w_{i}\right\}=\left\{p_{r}\right\}
\end{array}
$$

where:

$[\mathrm{H} 1]$... is the matrix of coefficients of the function $\frac{1}{\xi}$

$[H 2] \ldots$ is the matrix of coefficients of the function $\frac{1}{\xi^{2}}$

$[\mathrm{H} 3]$... is the matrix of coefficients of the function $\frac{1}{\xi^{3}}$ 


$$
\begin{aligned}
& {[\mathrm{H} 101]=[\mathrm{H} 1][\mathrm{A01}]} \\
& {[\mathrm{H} 103]=[\mathrm{H} 1][\mathrm{A03}]} \\
& {[\mathrm{H} 202]=[\mathrm{H} 2][\mathrm{AO2}]} \\
& {[\mathrm{H} 301]=[\mathrm{H} 3][\mathrm{A01}]}
\end{aligned}
$$

The highest derivative in the first of equations (19) is of order 2 , so the number of algebraic equations is $\mathrm{N}-1$ equations. The highest derivative in the second of equations (19) is of order 4 , so the number of algebraic equations is $\mathrm{N}-3$ equations. The total number of algebraic equations is $2 \mathrm{~N}-4$ along with 6 boundary conditions at $\xi=0$ and $\xi=1$, leading to $2 \mathrm{~N}+2$ equations in $2 \mathrm{~N}+2$ unknowns, which can be easily solved.

It is finally important to note that all matrices in the first of equations (19) are of order $(\mathrm{N}-1 \times \mathrm{N}+1)$, while all matrices in the second of equations (19) are of order ( $\mathrm{N}-3$ $\mathrm{xN}+1)$.

\section{Boundary Conditions}

Fortunately, it is relatively easy to represent any shell boundary conditions for the functions expanded in Chebyshev series.

\section{Top-vertex conditions}

At the vertex $\xi=0$ :

1. The displacement perpendicular to the axis of the shell is zero because of axisymmetry, which leads to:

$\mathrm{u} \sin \alpha+\mathrm{w} \cos \alpha=0$

2. $\frac{d w}{d \xi}=0$ for finite $M_{x}$ and $M_{\theta}$

3. The vertical displacement $(-u \cos \alpha+w \sin \alpha)$ at the vertex is a maximum, which leads to: $\frac{d u}{d \xi}=0$

\section{Clamped-edge conditions}

\section{Hinged-edge conditions:}
1. $u=0$
1. $u=0$
2. $w=0$
2. $w=0$
3. $\frac{d w}{d \xi}=0$
3. $\mathrm{M}_{\mathrm{x}}=0$ i.e. $\frac{d^{2} w}{d \xi^{2}}+\frac{v}{\xi} \frac{d w}{d \xi}=0$

The previous sets of boundary conditions can be written in matrix form by calculating the function or its derivatives at $\xi=0$ or $\xi=1$ as follow: 


\section{Vertex boundary conditions:}

At $\xi=0$

1. $\sin \alpha[T R O]\left\{u_{i}\right\}+\cos \alpha[T R O]\left\{W_{i}\right\}=0$

2. 4 [TR01] [A01] $\left\{W_{i}\right\}=4\left[\right.$ TTOAO1] $\left\{W_{i}\right\}=0$

3. 4 [TRO1] [A01] $\left\{u_{i}\right\}=4\left[\right.$ TOAO1] $\left\{u_{i}\right\}=0$

where:

[TR0] is a row matrix of $\mathrm{N}+1$ Chebeychev terms at $\xi=0$

[TR01] is a row matrix of $N$ Chebeychev terms at $\xi=0$

Note: the first term of Chebeyshev terms must be halved.

\section{Clamped-base boundary conditions:}

At $\xi=1$
1. $[T R 1]\left\{u_{i}\right\}=0$
2. [TR1] $\left\{W_{i}\right\}=0$
3. 4 [TR11] [A01]) $\left.\left\{W_{i}\right\}=4[T 1 A 01]\right)\left\{W_{i}\right\}=0$

where:

[TR1] is a row matrix of $\mathrm{N}+1$ Chebeychev terms at $\xi=1$

[TR11] is a row matrix of $N$ Chebeychev terms at $\xi=1$

Note: the first term of Chebeyshev terms must be halved.

\section{Hinged-edge conditions:}

At $\xi=1$

1. [TR1] $\left\{u_{i}\right\}$

2. [TR1] $\left\{W_{i}\right\}=0$

3. $(16[\mathrm{TR} 12][\mathrm{A02}]+4[\mathrm{TR} 11][\mathrm{A01}])\left\{\mathrm{W}_{\mathrm{i}}\right\}=(16[\mathrm{~T} 1 \mathrm{A02}]+4[\mathrm{~T} 1 \mathrm{A0} 1])\{\mathrm{Wi}\}=0$

where:

[TR11] is a row matrix of $\mathrm{N}$ Chebeychev terms at $\xi=1$

[TR12] is a row matrix of $\mathrm{N}-1$ Chebeychev terms at $\xi=1$

Note: the first term of Chebeyshev terms must be halved.

\section{RESULTS AND DISCUSSIONS}

\section{Problem 1:}

A complete cone with clamped base under uniform internal pressure p, Fig. 3 .

Material:

$E=3010^{6}$ psi $(206.85 \mathrm{GPa})$;
Geometry:

$L / h=50$;

$\alpha=45$ 
The functions $\frac{1}{\xi}, \frac{1}{\xi^{2}}$ and $\frac{1}{\xi^{3}}$ appearing in equation (18) are forced to take on their actual values at 10 points other than $\xi=0$. The system of equations(19), (20) and (21) are solved and the coefficients $\left\{u_{i}\right\}$ and $\left\{W_{i}\right\}$ are obtained. The strain and curvature components $\varepsilon_{\theta}$ and $k_{\theta}$ are computed at $\xi=0.001$ to avoid the singularity at $\xi=0$.

Table 1 shows the results of a convergence study with regard to $\overline{N x}(2 \mathrm{Nx} / \mathrm{pl} \tan \alpha)$ and $\overline{N \theta}(2 \mathrm{~N} \theta / \mathrm{p} \mid \tan \alpha)$ along the generator. It can be seen that the solution convergence is good, and that fairly accurate results can be obtained with 20 terms in the Chebyshev series. Table 2 and Figs. 5-8 compare the computed results $\overline{N x}, \overline{N \theta}$, $\overline{M x}\left(200 \mathrm{M}_{\mathrm{x}} / \mathrm{pL^{2 }} \tan (\alpha)\right)$ and $\overline{M \theta}\left(200 \mathrm{M}_{\theta} / \mathrm{p} \mathrm{L}{ }^{2} \tan (\alpha)\right)$ using 18 terms with the exact results obtained in reference [2] using Kelvin functions. It is seen that the computed results are very close to the exact ones with an average error less than $1.85 \%$

\section{Problem 2:}

A truncated frustum under a line load normal to the surface, and a horizontal line load at the base, Fig. 4. The upper edge is free, while the lower base is supported on rollers.

\section{Material:}

$\mathrm{E}=200 \mathrm{Gpa} \quad v=.3$

\section{Geometry:}

$L=1 m \quad L 1=0.4226497 m \quad L p=0.5381198 m \quad \alpha=30^{\circ}$

\section{Applied load:}

$$
P=1000 \mathrm{~N} / \mathrm{m} \quad H_{f}=-100 \mathrm{~N} / \mathrm{m} \quad M_{x}=10 \mathrm{Nm} / \mathrm{m}
$$

A new non-dimensional parameter $\xi=\frac{x-L_{1}}{L}$ is used to make the upper edge lie at $\xi=0$.

The system of equations (18) is applied except that the functions $\frac{1}{\xi}, \frac{1}{\xi^{2}}$ and $\frac{1}{\xi^{3}}$ become $\frac{1}{\left(\xi+\frac{L_{1}}{L}\right)}, \frac{1}{\left(\xi+\frac{L_{1}}{L}\right)^{2}}$ and $\frac{1}{\left(\xi+\frac{L_{1}}{L}\right)^{3}}$ respectively. Matrices [H1], [H2] and [H3] appearing in these equations are calculated for the new functions. The line load $P$ is 
distributed over $\Delta \xi=\xi_{2}-\xi_{1}$ to convert it into uniform pressure. Equation (4) is used to obtain the loading coefficients $p_{r}=\frac{2}{\pi} \int_{\xi 1}^{\xi 2} \frac{f(\xi) T_{r}(\xi)}{\sqrt{\xi} \sqrt{1-\xi}} d \xi ; 0 \leq \mathrm{r} \leq \mathrm{N}$.

\section{Boundary conditions:}

\section{Free-edge conditions:}

\section{Roller-supported-edge conditions:}

At $x=L_{1}$ i.e. $\xi=0$

1- $N_{x}=0$

2- $Q_{x}=0$

3- $M_{x}=10$

$$
\text { At } x=L_{2} \text { i.e. } \xi=1
$$

1- Vertical displacement $(-u \cos \alpha+w \sin \alpha)=0$

2- $M_{\mathrm{x}}=0$

3- Horizontal force $\left(N_{x} \sin \alpha+Q_{x} \cos \alpha\right)=-100$

The results are computed and compared with the exact solution obtained in reference [14] using Green's function. Table 3 and Figs. (9-12) compare the distributions of the normal displacement $w$ and the stress resultants $N_{x}, M_{x}$, and $Q_{x}$. The normal displacement $w$ and the axial force $N_{x}$ are calculated using 20 Chebebyshev terms, while the transverse shear $Q_{x}$ and Longitudinal moment $M_{x}$ are calculated using 70 terms because of the discontinuity at the point of load application. The table and figures show good agreement between the results with small discrepancy around the singularity.

\section{CONCLUSION}

A technique is presented for the solution of boundary-value problems described by a system of simultaneous differential equations with variable coefficients by expressing the unknown functions in terms of Chebeyshev series. A new matrix formulation is presented for the technique, which systematically transforms the problem into a system of algebraic equations, which can be readily solved in a short time on the computer.

The suggested technique is applied to two problems of thin, bending-resistant conical shells with different boundary conditions and external loads. Resuits are compared with the exact ones, and good agreement is found between the results. The applications prove that the suggested technique is accurate and easily applicable to difficult boundary-value problems. 


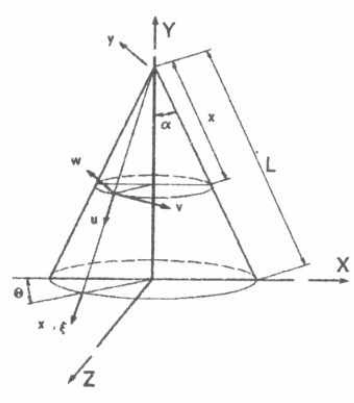

Fig. 1 Conical Shell Geometry

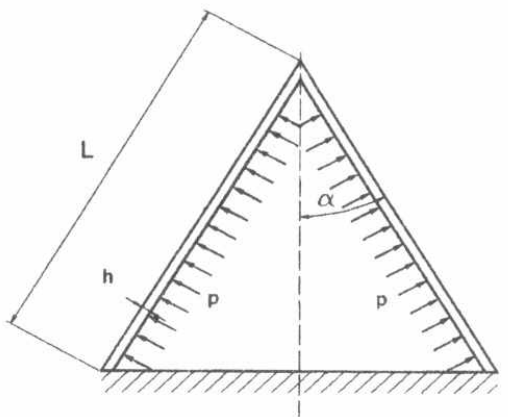

Fig. 3 Pressurized conical shell with clamped edge

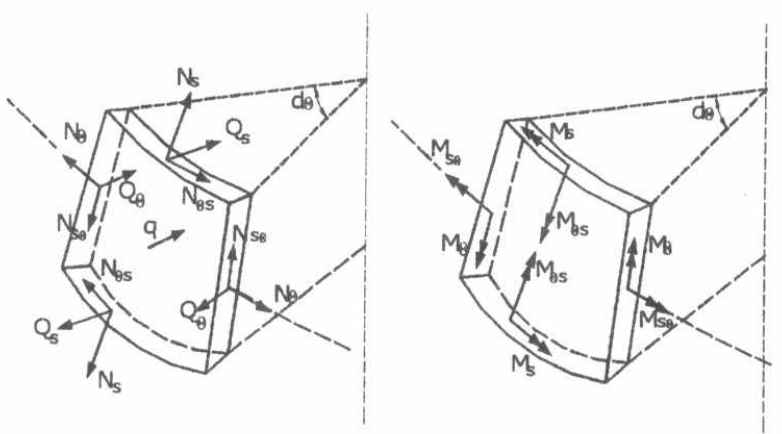

Fig. 2 Shell Stress Resultants

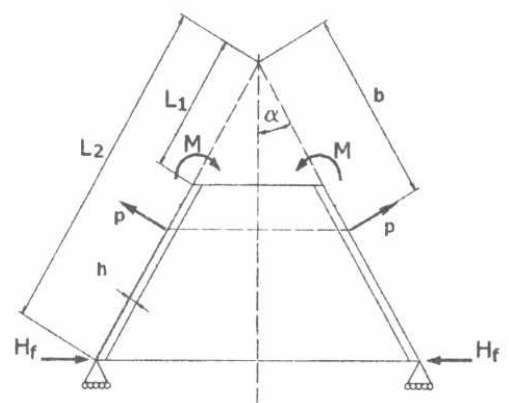

Fig. 4 Truncated cone under line loads

Table 1 Convergence Study Of Conical Shell Clamped At Its Base. $\overline{N x}$

\begin{tabular}{|c|c|c|c|c|c|c|c|c|c|}
\hline$\xi$ & 0.2 & 0.3 & 0.4 & 0.5 & 0.6 & 0.7 & 0.8 & 0.9 & 1 \\
\hline 16 & 0.1988 & 0.3034 & 0.399 & 0.5 & 0.602 & 0.715 & 0.815 & 0.857 & 0.823 \\
\hline 18 & 0.2037 & 0.2996 & 0.401 & 0.498 & 0.604 & 0.713 & 0.816 & 0.857 & 0.823 \\
\hline 20 & 0.2030 & 0.3014 & 0.399 & 0.499 & 0.603 & 0.714 & 0.817 & 0.857 & 0.823 \\
\hline
\end{tabular}

$\overline{N \theta}$
\begin{tabular}{|l|l|l|l|l|l|l|l|l|l|}
\hline 16 & 0.3850 & 0.617 & 0.777 & 1.012 & 1.252 & 1.491 & 1.471 & 0.857 & 0.246 \\
\hline 18 & 0.4025 & 0.5957 & 0.798 & 0.999 & 1.254 & 1.495 & 1.467 & 0.858 & 0.246 \\
\hline 20 & 0.3994 & 0.6004 & 0.794 & 1.002 & 1.252 & 1.496 & 1.467 & 0.858 & 0.246 \\
\hline
\end{tabular}




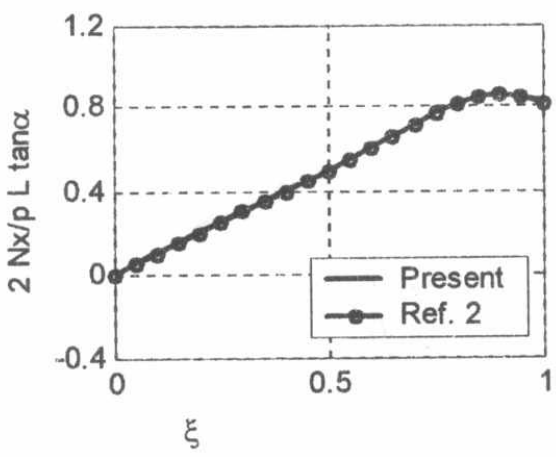

Fig. 5 Axial-force distribution

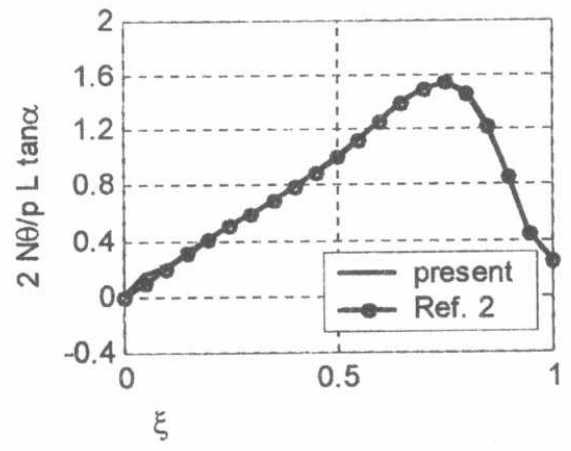

Fig. 6 Circumfrential-force distribution

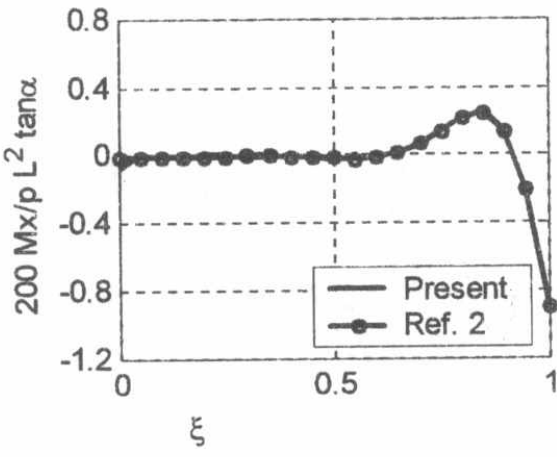

Fig 7 Axial-moment distribution

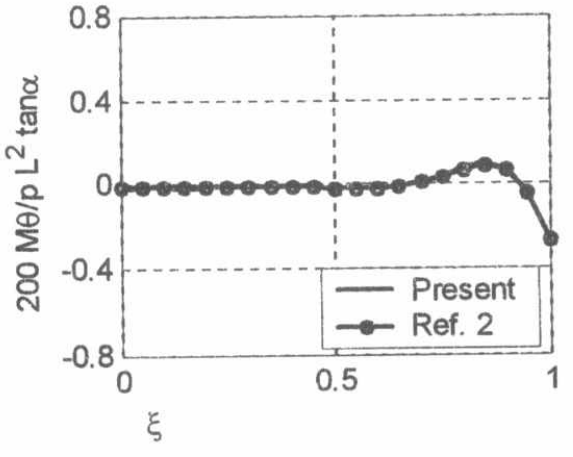

Fig. 8 Circumfrential-moment distribution

Table 2 Comparison of computed results $(\mathrm{N}=20)$ of complete cone with the exact ones.

\begin{tabular}{|c|c|c|c|c|c|c|c|c|}
\hline \multirow{2}{*}{$\xi$} & \multicolumn{2}{|c|}{$\overline{N x}$} & \multicolumn{2}{c|}{$\overline{N \theta}$} & \multicolumn{2}{c|}{$\overline{M x}$} & \multicolumn{2}{c|}{$\overline{M \theta}$} \\
\cline { 2 - 9 } & Present & Exact & Present & Exact & Present & Exact & Present & Exact \\
\hline 0.2 & 0.2031 & 0.2000 & 0.3995 & 0.4003 & -0.0134 & -0.0143 & -0.0143 & -0.0143 \\
\hline 0.3 & 0.3014 & 0.3001 & 0.6004 & 0.5995 & -0.0132 & -0.0134 & -0.0138 & -0.0139 \\
\hline 0.4 & 0.3999 & 0.3994 & 0.7941 & 0.7948 & -0.0161 & -0.0153 & -0.0146 & -0.0144 \\
\hline 0.5 & 0.4997 & 0.4987 & 1.0024 & 1.0020 & -0.0246 & -0.0255 & -0.0183 & -0.0185 \\
\hline 0.6 & 0.6034 & 0.6028 & 1.2525 & 1.2530 & -0.0241 & -0.0231 & -0.0199 & -0.0196 \\
\hline 0.7 & 0.7141 & 0.7143 & 1.4966 & 1.4964 & 0.0562 & 0.0565 & 0.0075 & 0.0076 \\
\hline 0.8 & 0.817 & 0.8149 & 1.4677 & 1.4635 & 0.2154 & 0.2146 & 0.0717 & 0.0717 \\
\hline 0.9 & 0.8573 & 0.8585 & 0.8589 & 0.8573 & 0.1341 & 0.1343 & 0.0685 & 0.0684 \\
\hline 1.0 & 0.8233 & 0.8207 & 0.2470 & 0.2462 & -0.8936 & -0.8968 & -0.2681 & -0.2690 \\
\hline
\end{tabular}




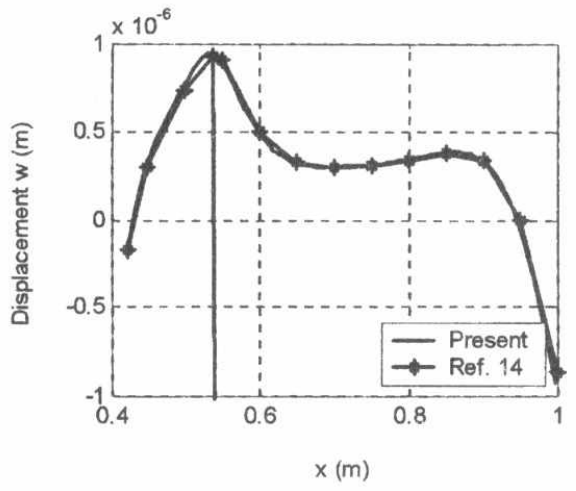

Fig. 9 Normal displacement $w$ (frustum)

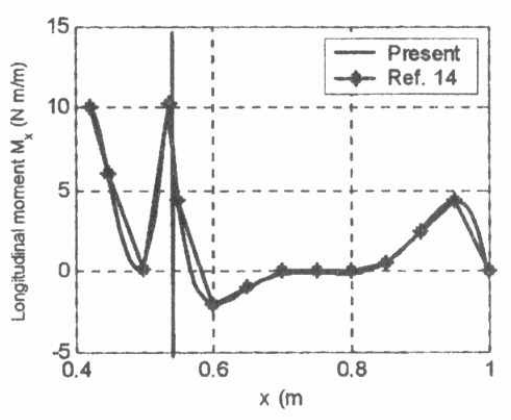

Fig. 11 Bending-moment distribution (frustum)

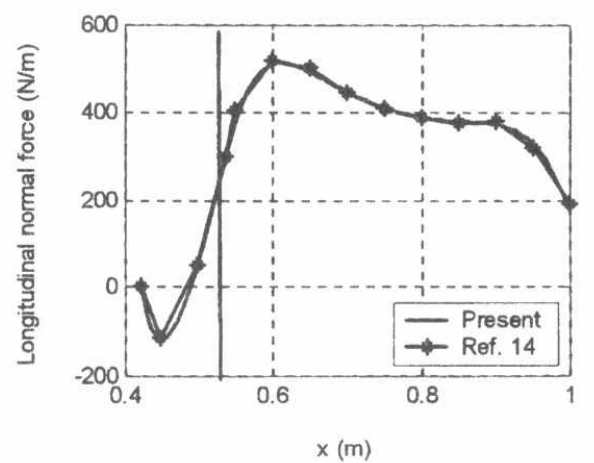

Fig. 10 Axial force distribution (frustum)

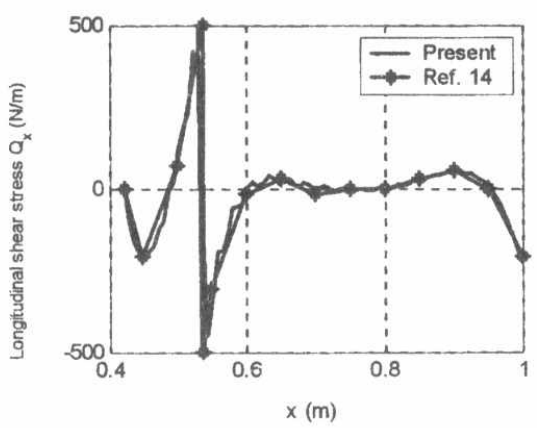

Fig. 12 Shear-force distribution (frustum)

Table 3 Comparison of computed results $(\mathrm{N}=70)$ of a frustum with the exact ones.

\begin{tabular}{|c|l|l|l|r|l|r|r|c|}
\hline \multirow{2}{*}{$\xi$} & \multicolumn{2}{|c|}{ Wx $10^{-6}$} & \multicolumn{2}{c|}{$N x$} & \multicolumn{2}{c|}{$M x$} & \multicolumn{2}{c|}{$Q x$} \\
\cline { 2 - 9 } & Present & Exact & Present & Exact & Present & Exact & Present & Exact \\
\hline 0.6 & 0.4921 & 0.3427 & 509.94 & 517.89 & -2.1947 & -2.096 & -13.48 & -13.92 \\
\hline 0.7 & 0.3017 & 0.3258 & 446.58 & 442.72 & -0.1805 & -0.149 & 1.34 & 1.41 \\
\hline 0.8 & 0.3462 & 0.3469 & 388.72 & 390.71 & -0.1202 & -0.181 & 2.91 & 2.764 \\
\hline 0.9 & 0.3384 & 0.3386 & 373.15 & 374.56 & 2.4440 & 2.453 & 49.07 & 50.84 \\
\hline 1.0 & -0.9361 & -0.937 & 182.149 & 182.99 & -0.0000 & 0.001 & -220.63 & -221.15 \\
\hline
\end{tabular}




\section{REFERENCES}

1. Timoshenko, S. P. and Woinowsky-Krigger, S. Theory of plates and shells, $2^{\text {nd }}$ ed., Mc-Graw Hill Book Co., New York, 1959

2. Harry Kraus, Thin Elastic Shells, John Wiley \& sons, Inc., New York. London. Sydney, 1967.

3. Geckeler, J. W., "Über die Festigkeit achensymetrischer Schalen," Forschungsarb. Ingwes., Berlin, 276, 1-52 (1926).

4. Langer, R. E., "On the Asymptotic Solution of Ordinary Differential Equations." Trans. Am. Math. Soc., 37, 397-416 (1935).

5. Forsythe, G. E., and Wasow, W. R., Finite Difference Methods for Partial Differential Equations, New York: John Wiley (1960).

6. Penny, R. K., "Symmetric Bending of the General Shell of Revolution by Finite Difference Methods, J. Mech. Eng. Sci., 3, 369-377 (1961)

7. Hubka, R. E., "A Generalized Finite Difference Solution of Axisymmetric Stress States in Thin Shells of Revolution," Report EM-11-19, Space Technology Laboratories Los Angeles, Calif. (1961).

8. Meyer, R. R., and Harmon, M. B., "Conical Segment Method for Analyzing Open Crown Shells of Revolution for Edge Loading," AIAA J., 1, 886-891 (1963)

9. T. Kant and M. P. Menon, Estimation of interlaminar stresses in fiber reinforced composite cylindrical shells, Computers \& Structures. Vol. 38, No. 2, pp. 131-147, 1991.

10. Galletly, G. D., " Influence Coefficients for Open Crown Hemispheres," Trans. A. S. M. E., 82A, 73-81 (1960).

11. Kalnins, A., "Analysis of Shells of Revolution Subjected to Symmetrical and Nonsymmetrical Loads," J. Appl. Mech., 31, 467-476 (1964)

12. R. S. Alwar and M. C. Narasimhan, Application of Chebyshev polynomials to the analysis of laminated axisymmetric spherical shell. Comps. Struct. 15, 215-237, 1990.

13.R. S. Alwar and M. C. Narasimhan, Analysis of laminated orthotropic spherical shells subjected to asymmetric loads. Computer \& Structures vol. 41, No. 4, pp. 611-620, 1991.

14.S. A. Tavares, Thin conical shells with constant thickness and under axisymmetric load. Computer \& Structures vol. 60, No. 6, pp. 895-921, 1996. 